\title{
Micropower High-Detectivity Infrared Sensor System
}

\author{
David T. Chang, Gladney C. Asada, William J. Kaiser, Oscar M. Stafsudd \\ Electrical Engineering Department, University of California, Los Angeles \\ Los Angeles, CA 90095-1594
}

\begin{abstract}
In this paper we report a thermal infrared detector system for wireless integrated network sensors (WINS). This detector system includes a high sensitivity thin-film radiation thermopile and a micropower analog-to-digital converter (ADC) optimized for this unique system. The thermopile has an excellent responsivity of $100 \mathrm{~V} / \mathrm{W}$ and a normalized detectivity of $1.1 \times 10^{9} \mathrm{~cm}^{*} \mathrm{~Hz}^{1 / 2} / \mathrm{W}$ in vacuum. The $\mathrm{ADC}$ includes a chopper for low noise measurement of the low frequency infrared sensor output. The ADC provides greater than 9-bit resolution and DC stability at a micropower level of $30 \mu \mathrm{W}$.
\end{abstract}

\section{Introduction}

The development and deployment of tactical sensors and distributed monitoring and control systems have been hindered in the past by the requirements of complex installation and communication network requirements. Conventional distributed sensors have required cable interface, and therefore, extensive modification to structures for sensor installation. The wireless integrated network sensor (WINS) technology, reported here, provides new product opportunities and new system capabilities.

Low power systems offer a new approach for distributed sensors based on a wireless sensor infrastructure. A wireless microsensor network may be distributed rapidly and without modification to large structures and systems. Also, wireless sensors may be applied in areas where volume and mass constraints limit the application of conventional wireline interface sensors. The wireless network architecture allows microsensor nodes to be deployed in a broad spectrum of commercial and military applications ranging from clinical medicine, precision manufacturing, and transportation to battlefield perimeter security and shoreline reconnaissance. Wireless microsensor nodes may also be applied to rotating machinery without the complex slipring systems that would normally be required for a conventional sensor electrical interface.

A large class of WINS applications will operate with battery energy sources at micropower levels [1]. For example, typical low duty cycle, low peak data rate (1kbps) and short range $(10-30 \mathrm{~m})$ communication can permit $30 \mu \mathrm{A}$ average current for a WINS node operating at $3 \mathrm{~V}$. A conventional, $(2.5 \mathrm{~cm}$ diameter, $0.7 \mathrm{~cm}$ thickness) $\mathrm{Li}$ coin cell provides this current and bias level for greater than three years of unattended operating life.

WINS applications in manufacturing, medical, and tactical areas bring new requirements for infrared pyrometry and imaging. The stringent power consumption requirement of $100 \mu \mathrm{W}$ dissipation and three-year operation with lithium coin cell places restriction on the selection of infrared sensors to be used in wireless applications. Since they require no bias current for operation, thermopiles and pyroelectric [2] infrared detectors are favored over bolometer detectors. Now, pyroelectric sensors provide the required high frequency performance. However, battlefield and commercial applications demand operation at low frequency. Further, due to the poor detector gain at low frequency, pyroelectric sensors due not provide adequate DC response capability. Thus, for new WINS applications, thermopile technology has been selected for the thermal infrared detector.

Thermal infrared sensor design is constrained by the limitations induced by electronic and thermal noise sources. Thermal noise power, determined by thermal conductance of the supports for the infrared absorbing element, increases with increasing conductance of the sensor structure. The ideal thermopile detector would be supported by mechanical structures with thermal conductances no greater than that of the thermopile itself. Now, electronic Johnson noise increases with increasing electrical resistance of the structure. The optimal combination of materials for thermopile applications, therefore, depends on thermoelectric coefficient, and electronic and thermal conductances. The figure of merit of the material of choice is, $Z=\alpha^{2} /(\rho * \kappa)$ where $\alpha$ is the thermoelectric power, $\rho$ the electrical resistivity, and $K$ the thermal conductivity.

\begin{tabular}{|l|c|c|c|l|}
\hline Element & $\begin{array}{c}\alpha \\
(\mu \mathrm{V} / \mathrm{K})\end{array}$ & $\begin{array}{c}\rho \\
(\mu \Omega \mathrm{m})\end{array}$ & $\begin{array}{c}\kappa \\
\left(\mathrm{W} / \mathrm{m}^{*} \mathrm{~K}\right)\end{array}$ & $\begin{array}{c}\mathrm{Z} \\
\left(10^{-6} / \mathrm{K}\right)\end{array}$ \\
\hline Antimony & 32 & 0.417 & 18.9 & 130 \\
\hline Bismuth & -72.8 & 1.1 & 8.38 & 574 \\
\hline Iron & 13.4 & 0.086 & 79 & 26 \\
\hline Nickel & -20.4 & 0.0614 & 90 & 75 \\
\hline p-poly Si & 400 & 10 & 84 & 190 \\
\hline n-poly Si & -400 & 10 & 84 & 190 \\
\hline
\end{tabular}

It is seen that the antimony-to-bismuth thermocouple junction system is ideal for this application.

In addition to requirements on sensor sensitivity and stability, the WINS system requires a micropower data converter interface for the thermopile sensor. Design challenges for this data converter include the increase in input referred noise resulting from power reduction. In addition, WINS infrared detector applications require low noise performance at low frequency (less than $10 \mathrm{~Hz}$ ) for surveillance and condition based maintenance. This creates additional challenges since CMOS interface circuits display large " $1 / \mathrm{f}$ " noise, yielding unacceptable low frequency performance. The $\Sigma-\Delta$ converter described here, designed for operation with the thermopile infrared sensor, incorporates an input chopper, providing DC stability in micropower CMOS.

\section{Thermopile Fabrication}

Thermal infrared sensors rely on a temperature sensitive element that may absorb incident radiation and is thermally isolated from its fixed temperature support. Sensitivity, limited by thermal noise contributed by the finite thermal conductance of the support, is optimized by reducing thermal conductance of the structure. For this sensor, a thin film insulating membrane 
supports the thermopile structure. The thin-film radiation thermopile infrared detector is created by the conventional bulk micromachining method. The device is fabricated as follows (figure 1). First, a $0.5 \mu \mathrm{m}$ thickness of LPCVD low-stress nitride is deposited onto an n-type silicon substrate to form the structural layer. By using front and backside wafer photolithography and reactive ion etching, etch windows and alignment marks are created. Anisotropic etching of the silicon substrate in $30 \%$ potassium hydroxide $(\mathrm{KOH})$ solution at $80^{\circ} \mathrm{C}$ for 4 hours leaves a $30 \mu \mathrm{m}$ thick silicon layer behind the silicon nitride membrane for simplification of the subsequent processing. The detector element consists of 30 pairs of bismuth to antimony measurement junctions on the silicon nitride membrane and an equal number of reference junctions on the silicon frame. The $24 \mu \mathrm{m}$ wide, $0.3 \mu \mathrm{m}$ thick bismuth and antimony lines are deposited by a single-step optical photolithographic lift-off process with chlorobenzene treatment.

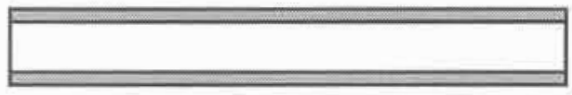

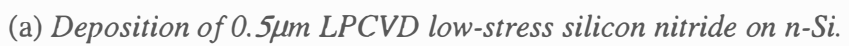

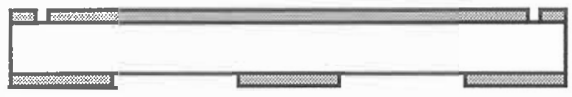

(b) Front and back lithography and RIE backside etch windows.

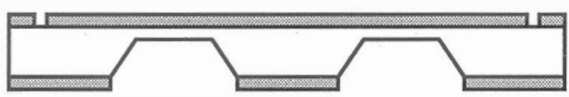

(c) $\mathrm{KOH}$ deep etch @ 80 C for 4 hours, leave $30 \mu \mathrm{m}$ Si.

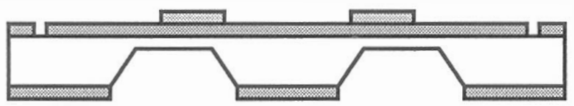

(d) E-beam evaporate bismuth thin film, acetone lift-off.

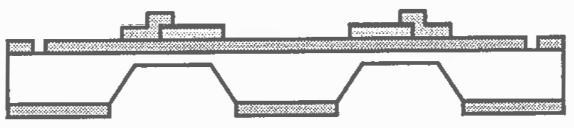

(e) E-beam evaporate antimony thin film, acetone lift-off.

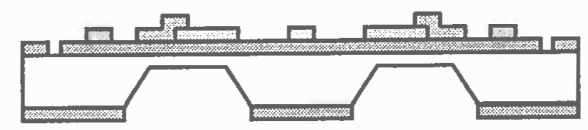

(f) E-beam evaporate titanium/gold thin films, acetone lift-off.

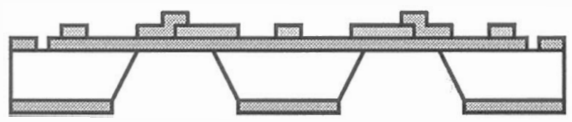

(g) Protect top side with wax, remove the last $30 \mu \mathrm{m}$ of $\mathrm{Si}$.

Figure 1. Bismuth/Antimony thermopile fabrication process flow.

Titanium and gold bonding pads are then evaporated to complete the thermopile. After all steps involving photolithography are

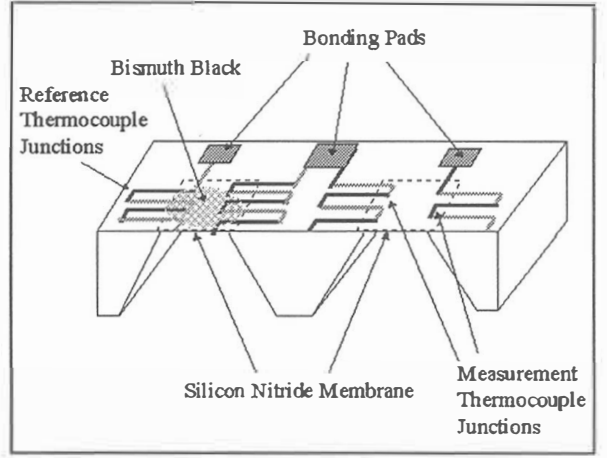

Figure 2. A simplified cross-section view of the dual-element 30thermocouple bismuth-antimony radiation thermopile.

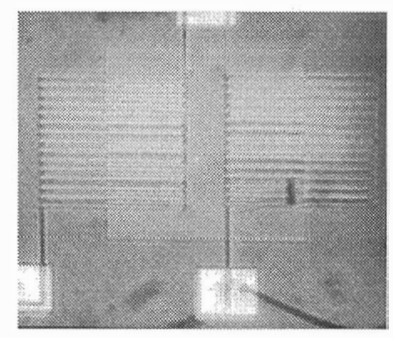

Figure 3. Top view of the bismuth-antimony radiation thermopile.

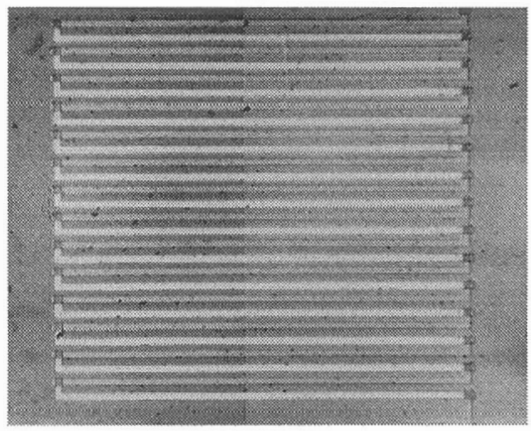

Figure 4. Top view of one half of the detector element. Reference junctions are on the silicon frame (left half) and the measurement junctions on the $0.5 \mu \mathrm{m}$ thick silicon nitride membrane (right half.)

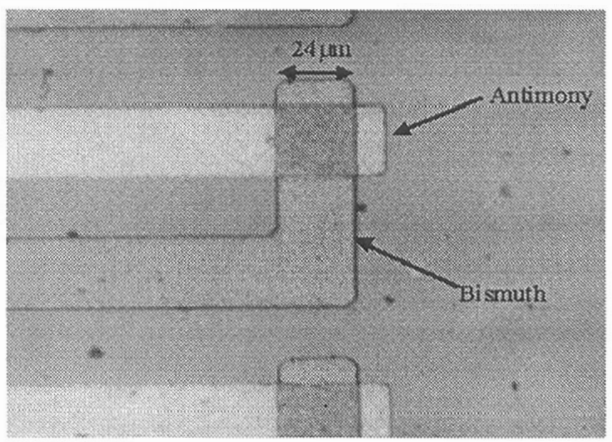

Figure 5. The bismuth-antimony thermocouple junction.

completed, the wafer is protected by wax on the device side and put back into $\mathrm{KOH}$ to remove the last $30 \mu \mathrm{m}$ of silicon behind the nitride membrane. In order to improve infrared absorption, a layer 
of metal black [2] is deposited at the center of the active area to complete the fabrication. A 3-D drawing and several top views of the detector are shown in figures 2-5.

\section{Micropower Analog-to-Digital Converter}

A micropower analog-to-digital converter (ADC) is required to enable digital signal processing of infrared signals for event detection. A first order Sigma-Delta $\left(\sum-\Delta\right)$ converter is chosen over other architectures because of power constraints. Architectures including parallel or pipelined ADCs provide high throughput at high power. Further, sequential approximation converters require multibit digital-to-analog converter (DAC) systems which also require large power dissipation. In contrast, the first order $\Sigma-\Delta$ converter uses a single integrator, a comparator, and a one bit DAC (figure 6).

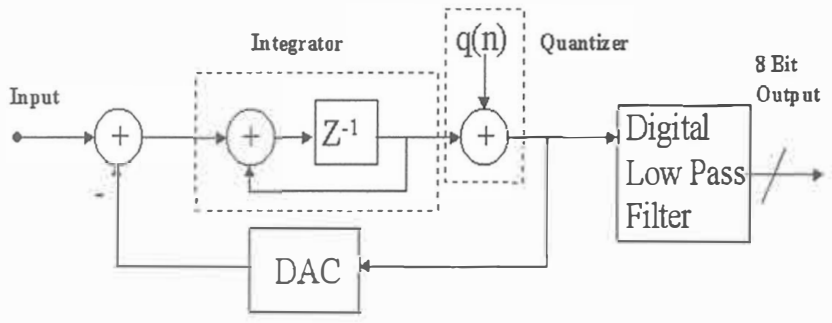

Figure 6. A block diagram of a first order Sigma-Delta analogto- digital converter loop.

Additional benefits appear as a result of the $\Sigma$ - $\Delta$ oversampled architecture. Specifically, by comparison, all other ADCs sample at the Nyquist rate of the input signal. Ensuring Nyquist rate signals requires the use of input anti-aliasing filters. However, the oversampled converter samples at a frequency $2^{\mathrm{N}}$ times greater than the Nyquist rate, where $\mathrm{N}$ is the resolution (word length) of the converter [3]. This characteristic translates the aliased input signal to a much higher frequency than in other architectures thus minimizing or eliminating the filter before the ADC.

A further benefit of the $\Sigma-\Delta$ architecture for micropower circuits is the ability to trade component requirements for the over sampling ratio (the sampling frequency divided by the Nyquist frequency). The analog components of the ADC operate in deep subthreshold to meet the goal of low current micropower operation [4]. This imposes severe restrictions on the performance of the circuits within the loop. A high oversampling ratio of 1024 is thus chosen to overcome the problems associated with low performance circuits. The possible increased power consumption of digital components in the signal path including the low pass filter is prevented with the use of low power cell libraries and architecture.

WINS infrared sensor applications present an additional challenge for ADC design. Specifically, the WINS infrared detector system must measure variations of infrared signals at low frequencies (less than $1 \mathrm{~Hz}$ ). This is required for continuous monitoring of a slowly moving threat object or monitoring of temperature variation in condition based maintenance applications

However, implementation of low noise ADC systems in CMOS encounters the severe " $1 / \mathrm{f}$ " input noise. Noise rejection and DC stability in these CMOS ADC systems is achieved here by signal "chopping" methods. The chopper ADC mixes the input signal to an intermediate frequency (IF) before delivery to the $\Sigma-\Delta$ loop. Another mixing stage, to bring the signal back into the band of interest, is done before the pulse code modulated signal is fed into the digital low pass filter (figure 7). This approach requires only the use of a commutating switch at the analog input (where no charge injection will occur) and a second commutating switch at the digital output, again where no charge injection error may occur.

Because of the low frequencies of the input signal, the main source of noise in the analog components of the ADC is $1 / \mathrm{f}$ noise. Moving the input signal to an IF minimizes the noise allowed to corrupt conversion [5]. The input of the $\Sigma-\Delta$ at an IF ensures that the loop will not saturate with a large DC output from the infrared sensor. Additional power consumption is avoided by the use of a passive mixer to chop the input to an IF of $1 / 8^{\text {th }}$ of the sampling frequency.

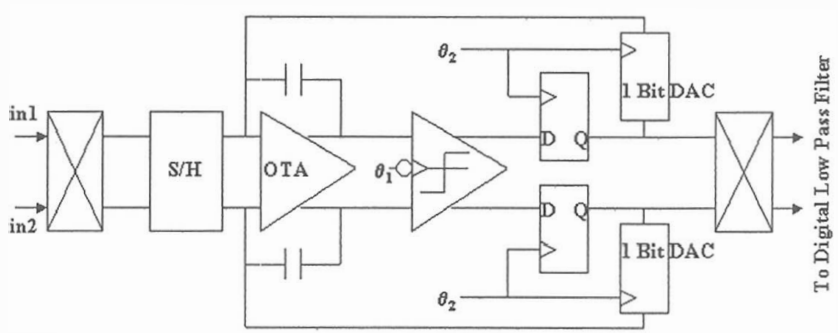

Figure 7. A block diagram of the pulse code modulator part of the $\Sigma \triangle A D C$ showing the location of the chopping blocks.

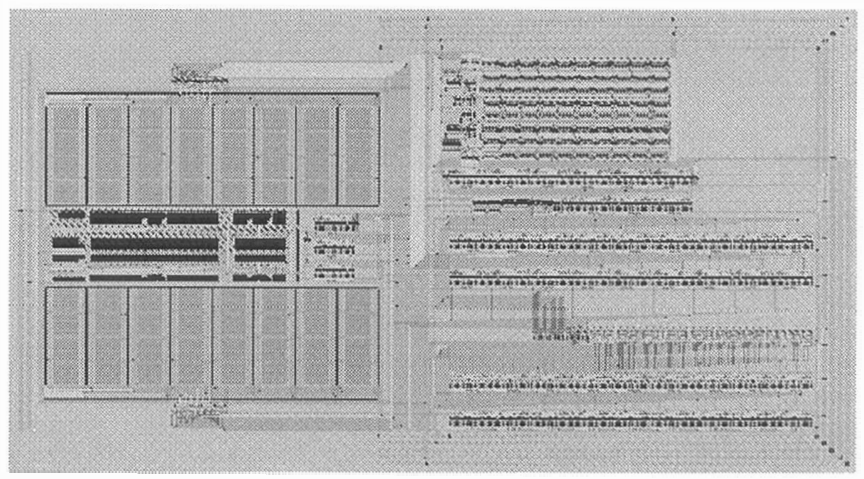

Figure 8. Layout of the core of the chopped $\Sigma \Delta A D C$ showing the analog components (on the left), the digital low pass filter (bottom right), and the clock generation circuit (top middle).

The first order $\Sigma-\Delta$ ADC has been fabricated in the HPCMOS $0.8 \mu \mathrm{m}$ process (figure 8 ). Direct measurement shows that the converter achieve greater than 9 bit resolution for a $100 \mathrm{~Hz}$ band limited signal with a power consumption of only $30 \mu \mathrm{W}$ on a single $3 \mathrm{~V}$ rail. This chopper ADC has been demonstrated to have a frequency-independent SNR from $0.1-100 \mathrm{~Hz}$. This resolution is adequate for the infrared sensor tactical motion detection application.

\section{Measurement Results}


The infrared detector characterization apparatus is shown in figure 9. A $1.55 \mu \mathrm{m}$ wavelength diode laser modulated by a function generator serves as the coherent infrared source.

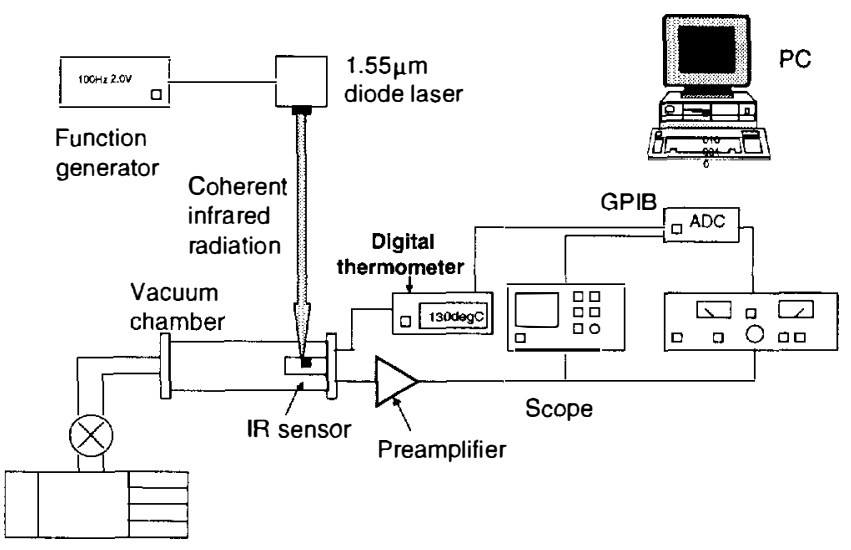

Figure 9. Infrared measurement setup for the thermopile detector.

The sensor can be tested in an evacuated environment to reduce thermal conduction loss to air. A scan of the infrared detector response is shown in figure 10.

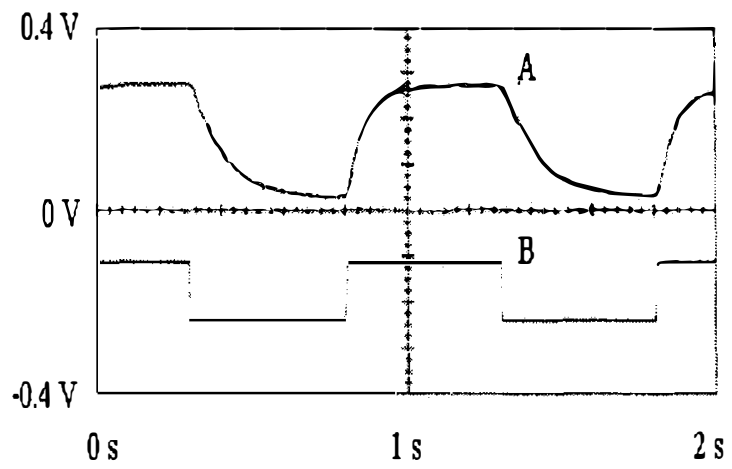

Figure 10. A scan of the infrared detector response in vacuum (trace A) to a $I \mathrm{~Hz}, 1.55 \mu \mathrm{m}$ wavelength, square-wave modulated radiation (trace $B$ ).

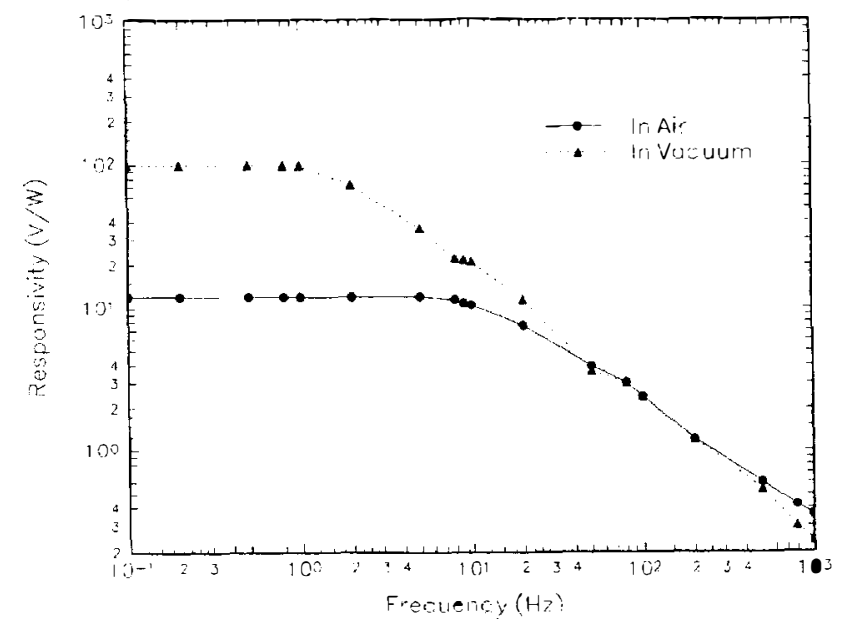

Figure 1I. Responsivity vs. frequency plot of the thermopile measured in air and vacuum. The influence of thermal conductance of the atmosphere is seen in the magnitude and frequency response.
In addition, the responsivity of the thermopile is measured as a function of frequency in both air and vacuum. Figure 10 shows that the response of the sensor in vacuum increases by almost an order of magnitude over that in air. The detector has a peak responsivity of $100 \mathrm{~V} / \mathrm{W}$ in vacuum and $12.5 \mathrm{~V} / \mathrm{W}$ in air. The noise-equivalent-power was measured by an HP35660A dynamic signal analyzer to be $1.8 \mathrm{nW} / \mathrm{Hz}^{1 / 2}$ at $1 \mathrm{~Hz}$. The normalized detectivity $D^{*}$ of this sensor is $1.1 \times 10^{9} \mathrm{~cm}^{*} \mathrm{~Hz}^{1 / 2} / \mathrm{W}$ in vacuum and $1.38 \times 10^{8} \mathrm{~cm}^{*} \mathrm{~Hz}^{1 / 2} / \mathrm{W}$ in air. This result compared favorably with a previously reported 60-thermocouple polysilicon infrared sensor [6]. This sensitivity is ideally suited for many of the applications listed above.

\section{CONCLUSIONS}

A micropower thermal infrared sensor based on suspended metal thermopile structures has been developed with a micropower CMOS data converter system. The thermopile sensor element is compatible with a range of integration technologies including flipchip technology methods. The development of a chopper $\Sigma-\Delta$ data converter provides DC stability. The detectivity of this detector is $1.1 \times 10^{9} \mathrm{~cm}^{*} \mathrm{~Hz}^{1 / 2} / \mathrm{W}$ in a vacuum package. Early applications of these devices will be with the WINS node platform for situational awareness. However, additional applications will follow in condition based maintenance.

\section{ACKNOWLEDGMENTS}

This research is supported by the Defense Advanced Research Projects Agency (DARPA). The authors would like to thank Pamela Patterson for valuable discussions.

\section{REFERENCES}

[1] K. Bult, A. Burstein, D. Chang, M. Dong, M. Fielding, E. Kruglick, J. Ho, F. Lin, T. H. Lin, W. J. Kaiser, H. Marcy, R. Mukai, P. Nelson, F. Newburg, K. S. J. Pister, G. Pottie, H. Sanchez, O. M Stafsudd, K. B. Tan, C. M. Ward, S. Xue, J. Yao," Technical Digest of the 1996 Solid-State Sensor and Actuator Workshop, pp. 205-210, 1996.

[2] D. T. Chang, D. M. Chen, F. H. Lin, W. J. Kaiser, O. M. Stafsudd, "CMOS Integrated Infrared Sensor," Proceedings of the 1997 International Conference on Solid-State Sensors and Actuators (Transducers '97), vol. 2, pp. 1259-1262, 1997.

[3] E. Vittoz, Design of Analog-Digital VLSI Circuits for Telecommunications \& Signal Processing. ed. J. E. Franca and Y. Tsividis, New York, Prentice Hall, 1994.

[4] P. Aziz, H. Sorensen, J. Spiegel, IEEE Signal Processing Magazine, vol. 13, pp. 61-70, 1996.

[5] Y. Chang, C. Wu, T. Yu, "Chopper-Stabilized Sigma-Delta Modulator," Proceedings of the 1993 IEEE International Symposium on Circuits and Systems, pp. 1286-1289, 1993.

[6] M. Muller, W. Budde, R. Gottfried-Gottfried, "A Thermoelectric Infrared Radiation Sensor," Proceedings of the 1995 International Conference on Solid-State Sensors and Actuators (Transducers '95), pp 640-3, 1995. 